\title{
Laser Metal Deposition on-line Monitoring via Plasma Emission Spectroscopy and Spectral Correlation Techniques
}

\author{
Jose J. Valdiande, Jesus Mirapeix, Jaume Nin, Eloi Font, Carlos Seijas and Jose Miguel Lopez- \\ Higuera Senior Member, IEEE
}

\begin{abstract}
Plasma spectroscopic techniques focused on the analysis of the plasma background radiation have been studied to enable an efficient on-line monitoring of a laser metal deposition process. The influence of different process parameters and elements, such as laser power, process speed, powder feeding rate and different powder and substrate compositions has been analyzed by means of several experimental trials. The resulting cladding patch analyzes via visual inspection and macrographs have been correlated with their associated spectroscopic monitoring signals. These studies have indicated that on-line quality monitoring of the laser metal deposition process is feasible by means of the proposed solutions, avoiding the identification and use of plasma emission lines. The latter improves the computational performance and avoids, not only the identification of each emission line, but also their specific sensitivity to certain defects. Spectral correlation techniques have also been proposed for monitoring purposes, thus enabling a more quantitative analysis.
\end{abstract}

Index Terms - optical sensor, laser metal deposition, on-line monitoring, plasma spectroscopy, quality assurance, correlation techniques, macrographs

\section{INTRODUCTION}

$\mathrm{L}$ ASER cladding, also known as laser metal deposition $\Lambda$ (LMD), was developed by several companies such as Rolls Royce, Fiat or GM at the beginning of 1980s [1]. It is based on the use of a laser beam to form a melt pool on a metallic substrate. Metal alloy powders are fed into the pool during the process, thus being melted and forming a clad track that is fusion-bonded to the substrate.

The authors would like to thank the staff of TM COMAS and Autotech Engineering for their valuable help during the design, implementation and test of the monitoring system. Fundació CTM Centre Tecnològic is also acknowledged for the performance of the macrographs. This work was supported in part by the Project "Noves tecnologies de laser cladding per a processos de conformat" (RD15-1-0098) funded by ACCIO (Generalitat de Catalunya) via FEDER funds. This work was also supported by projects PID2019-107270RB-C21/ AEI / 10.13039/501100011033.

J. Mirapeix and .J.M. Lopez-Higuera and are with the Photonics Engineering Group (University of Cantabria), CIBER-bbn and IDIVAL, 39005, Santander, Spain.(e-mail: miguel.lopezhiguera@unican.es; jesus.mirapeix@unican.es)

J.J.. Valdiande is with the Photonics Engineering Group (University of Cantabria) and Sadiq Engineering S.L. (e-mail jose.valdiande@unican.es).

J. Nin and E. Font are with Talleres Mecánicos Comas S.L.U., Blanes, Girona (Spain) (email: tecnic@tmcomas.com; tecnic2@tmcomas.com)

C. Seijas is with Gestamp/Autotech Engineering Co., Castellbisbal, Barcelona (Spain) (email: cseijas@cn.gestamp.com).
This protection coating results in improved features such as an increased corrosion and wear resistance. Additional advantages lie in the reduced heat affected zone and level of dilution.

As happens in many other manufacturing processes like arc or laser welding, there are several process parameters influencing the final quality of the clad tracks: laser power, cladding speed, powder feeding rate, shielding gas flow rate and powder composition, among others. The complexity of the Physics involved and the traditional manual supervision and control of these parameters have given rise to the proposal of different on-line monitoring solutions.

Temperature monitoring of the process was one of the first approaches explored. Bi et al. proposed a system based on a pyrometer, a photodiode and a CCD camera, operating at different wavelengths, able to estimate the process temperature [2]. The associated signals showed correlation with process parameters such as laser power, powder feeding rate and LMD speed. The resulting temperature profiles also showed a good correlation with the dilution and dimension of clad tracks. A dual-color pyrometer able to estimate the melt pool temperature was also proposed [3]. That information was used to propose a closed-loop system able to work at a certain temperature by adjusting the laser power. Some works have also been focused on the use of cameras to estimate and control the melt-pool size by adjusting the laser power in real time [4].

Recently, plasma optical spectroscopy solutions have been proposed for LMD monitoring by several authors, taking advantage of the knowledge generated for a similar scenario: on-line welding quality monitoring. In this paper, we propose the use of an on-line LMD quality monitoring system based on plasma spectroscopy, analysis of the background radiation and spectral normalization and correlation techniques. This spectral processing solutions avoid the necessity of identifying the spectral emission lines, thus simplifying the computational requirements and making the system independent of the specific sensitivity of each species to certain LMD defects. Experimental trials performed in the framework of a research project for the automotive industry, in particular for the repair of tools involved in hot-stamping processes, will show the feasibility of the proposed solution. 


\section{ON-LINE MONITORING VIA PLASMA OPTICAL SPECTROSCOPY}

Plasma optical spectroscopy has been widely studied and proposed for welding quality on-line monitoring solutions in different industrial environments. The analysis of the captured plasma spectra, mainly the emission lines that can be associated with specific species participating in the process, gives rise to the generation of output monitoring parameters that have been clearly correlated with different defects. Typically, the following assumptions are assumed to apply the plasma spectroscopy theory [5]: optically thin plasma, local thermal equilibrium and McWhirther criterion. Within this context, the relative intensity of the emission line $I_{m n}$ can be related to the population of the upper state $N_{m}$ as follows:

$$
I_{m n}=N_{m} A_{m n} h \gamma_{m n}
$$

where $A_{m n}$ is the transition probability, and $h_{\gamma m}$ is the energy of such a transition.

Ferrara et al. demonstrated the correlation of the plasma electronic temperature $T_{e}$ with different perturbations on both laser and arc (TIG: Tungsten inert gas) welding processes, using Ar I and Fe I emission lines [6]. The Boltzmann-plot method has been commonly employed in this regard, requiring several emission lines from the same species to enable an accurate $T_{e}$ estimation. A simplified method involving only two emission lines from the same species has also been considered for monitoring purposes [7]:

$$
T_{e}=\frac{E_{m}(2)-E_{m}(1)}{k \ln \left[\frac{I(1) A(2) g_{m}(2) \lambda(1)}{I(2) A(1) g_{m}(1) \lambda(2)}\right]},
$$

where $I$ is the emission line intensity, $\lambda$ the associated wavelength, $A$ the transition probability, $g$ the statistical and $E_{m}$ the upper level energy.

These authors also demonstrated that the sensitivity to detect some defects depends on the particular species used to estimate $T_{e}$, which may compromise the performance of the monitoring system.

Regarding LMD applications, Bartkowiak performed one of the first explorations of plasma spectroscopy in this field [8]. Plasma spectra was acquired under different process conditions, i.e. modifying laser power and scan speed, where several CR emission lines were identified. Song and Mazumder studied the $\mathrm{Cr}$ composition using calibration curves and involving both plasma temperature and electron density calculations via $\mathrm{Cr}$ I and Fe I lines [9]. In a recent study, an improved performance in the composition estimation was achieved by using support vector regression trained with spectral line intensity ratios and the spectral integrated intensity, using in this case Al I and Ti I lines [10]. Song et al. also demonstrated that the line ratio technique can be used to indicate phase transformations changes in clad layers [5]. The ratio between a $\mathrm{Fe} \mathrm{I}$ absorption line and the adjacent background signal was also proposed to detect the onset of metallic bonding [11]. Additionally, they also determined the extent of dilution with the estimation of $T_{e}$ via Boltzmann plot and four Cr I emission lines. The feasibility of using the line- to-continuum method with $\mathrm{Ti}$ I lines to detect the appearance of isolated and systematic flaws was also demonstrated by Stutzman et al. [12]. Chen et al. related the relative intensity of several emission lines to some process parameters, like laser power [13]. They also used a statistical process control solution enabling the detection of different perturbations, such as changes in laser power, powder feed rate and transverse speed. Photodiodes have also been proposed for LMD monitoring purposes: two photodiodes at 520 (Cr I) and 530 $\mathrm{nm}$ (background radiation) were employed in [14] to generate a line-to-continuum monitoring profile showing a relationship with the final percent void of the resulting components.

Apart from these strategies mainly based on the identification and use of specific emission lines, some authors have proposed alternative monitoring solutions for welding monitoring also based on the analysis of the plasma spectra. Sibillano et al. proposed the use of the covariance mapping technique to correlate the influence of process speed in laser welding to the loss of alloying elements [15]. The plasma continuum signal was also proposed to generate output monitoring parameters showing a clear correlation with some arc-welding defects [16]. A colorimetric-based approach was also presented by Mirapeix et al. [17], where color temperature and HSL (hue, saturation and luminance) parameters were chosen to detect process perturbations in arc welding. Kisielewick et al. have recently analyzed the morphology of the plasma spectra in a LMD process, establishing a relationship between laser power and the intensity of continuum radiation [18].

A similar and simplified approach is based on the estimation of the plasma RMS signal [19]:

$$
S_{R M S}=\sqrt{\frac{1}{n} \sum_{i=0}^{n-1} x_{i}^{2}},
$$

where $x_{i}$ is the intensity of the ith pixel of the chosen spectrometer. $\mathrm{S}_{\mathrm{RMS}}$ is an estimation of the average intensity emitted by the plasma and it represents and indirect measurement of the plasma spectrum energy within the spectral range under consideration. It is therefore related to the process heat input.

The latter proposals show a common feature: emission lines to be found in the plasma spectra do not need to be identified. This implies some advantages, not only in terms of computational cost and complexity, but also concerning flexibility to adapt the monitoring to different materials and independence from the particular sensitivity of each species to various defects.

In this paper we propose a monitoring system for laser metal deposition based on plasma spectroscopy and, specifically, techniques avoiding the use of emission lines, such as spectral normalization and correlation analysis. Experimental trials demonstrate the feasibility of this approach to identify defective clad tracks associated with different process parameters and materials. 


\section{EXPERIMENTAL SETUP AND PRELIMINARY TESTS}

Figure 1 shows an image of the LMD process, where the input optics and the optical fiber of the monitoring system can be observed (all the experimental tests described in this work have been carried out in the facilities of TM Comas (Girona, Spain)). This monitoring system consisted of an optical $5 \mathrm{~mm}$ diameter collimator and two optical fibers $(200 \mu \mathrm{m}$ and 2 and 8 meters long) connected by an attenuator used to control the amount of radiation reaching the spectrometers. Three different spectrometers were considered during the tests: HR4000, HR2000+ and ECONIC, whose main characteristics are described in Table 1.
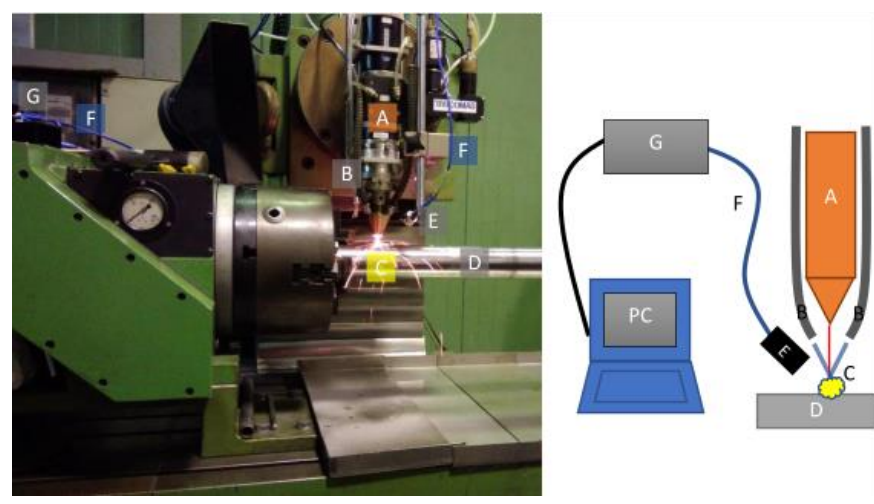

Fig. 1. Detail of the laser head and the attached input optics and optical fiber used during the experimental tests at TM Comas: (a) laser head, (b) powder and gas nozzles, (c) plasma, (d) substrate, (e) collimator, (f) optical fiber, (g) spectrometer.

TABLE I

MAIN CHARACTERISTICS OF SPECTROMETERS INVOLVED IN THE EXPERIMENTAL TESTS

Resolution (nm) $\quad$ Range (nm) $\quad$ Slit $(\mu \mathrm{m}) \quad$ Grating (lines)

\begin{tabular}{ccccc} 
HR4000 & 0.11 & $370-500$ & 5 & 1800 \\
HR2000+ & 1.1 & $200-1100$ & 5 & 300 \\
ECONIC & 1 & $200-850$ & 25 & 600 \\
\hline
\end{tabular}

A Laserline LDF 1000-4000 VG4L was used to produce the clad tracks, considering AISI-316 and QR0-90 as steel substrates and different powder compositions to analyze their effect on the resulting quality. The diameter of the laser spot on the surface of the base material is $2 \mathrm{~mm}$, with a focal distance of $14 \mathrm{~mm}$. Argon is used as shielding gas with a nominal flow rate of $20 \mathrm{l} / \mathrm{min}$, while the nominal feeding rate for the powders is $5 \mathrm{l} / \mathrm{min}$.

Initial tests were conducted to study the feasibility of a spectral analysis not based on the identification of emission lines. Figure 2 shows a comparison of the spectra associated with different laser powers (1000 and $2000 \mathrm{~W}$, Fig. 2(a)) and process speeds (400, 600 and $800 \mathrm{~mm} /$ minute, Fig. 2(b)). It can be observed how these spectra are clearly different, thus allowing a possible detection of defective situations, i.e. deviations from the established nominal parameters.
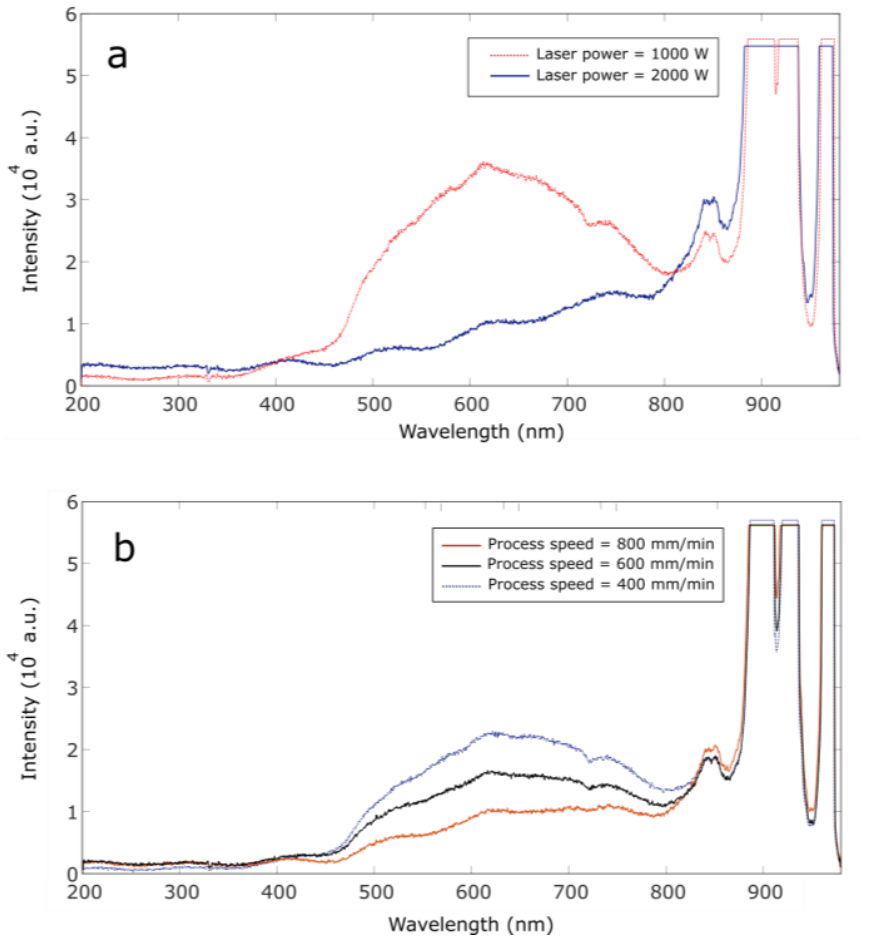

Figure 2. Spectra associated with AISI-316 as base material and different laser powers and process speeds: (a) laser powers of $1000 \mathrm{~W}$ (dotted line, red) and $2000 \mathrm{~W}$ (blue); (b) process speeds of 400 (dotted line, blue), 600 (black) and $800 \mathrm{~mm} / \mathrm{min}$ (red).
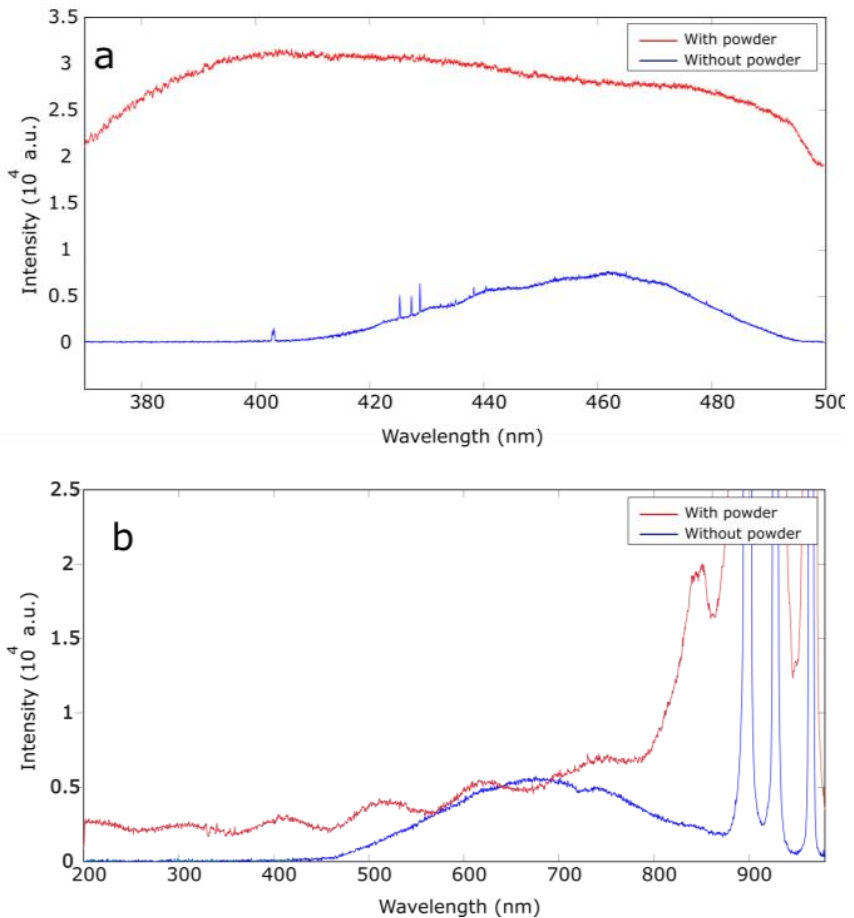

Figure 3. Comparison of spectra captured with different spectrometers during tests performed with QR0-90 as base material and with and without powder: (a) HR4000 spectrometer: with powder (red line) and without powder (blue line); (b) HR2000+ spectrometer: with powder (red line) and without powder (blue line). 
No clear emission lines can be observed in these spectra. This can be explained by the disposition of the input optics in terms of the laser focal point, which affects the relationship between the plasma emission lines and the background radiation as explained in [10] and [11]. Emission lines appear in some specific experimental situations, for example when the powder feeding rate is greatly reduced. Figure 3 (a) shows a comparison between spectra associated with clad tracks performed with constant powder feeding rate of $30 \mathrm{rpm}$ and without powder. In the latter scenario the chosen spectrometer (HR4000) established a spectral range from 370 to $500 \mathrm{~nm}$, with appearance of emission lines from 400 to $490 \mathrm{~nm}$. AISI316 was used as substrate material in Fig. 3 (a), while Fig. 3 (b) shows the same experiment using in this case QR0-90 as base material and a different spectrometer (HR2000+). Again, spectra from LMD with and without powder are clearly different, thus allowing a possible discrimination of these process situations.

Some process perturbations can be clearly detected with simple processing approaches. The evolution of a certain pixel $(1440, \lambda \approx 850 \mathrm{~nm})$ for two clad tracks performed on AISI-316 with powder feeding rates of 15 and $45 \mathrm{rpm}$ has been depicted in Figure 4 (a). The intensity and variance of the signal clearly vary, and it shows a clear lower stability in the second pass (the laser comes to the right end of the clad track and then comes back to the starting point). Figure 4(b) presents the associated clad tracks, which clearly show a different appearance.

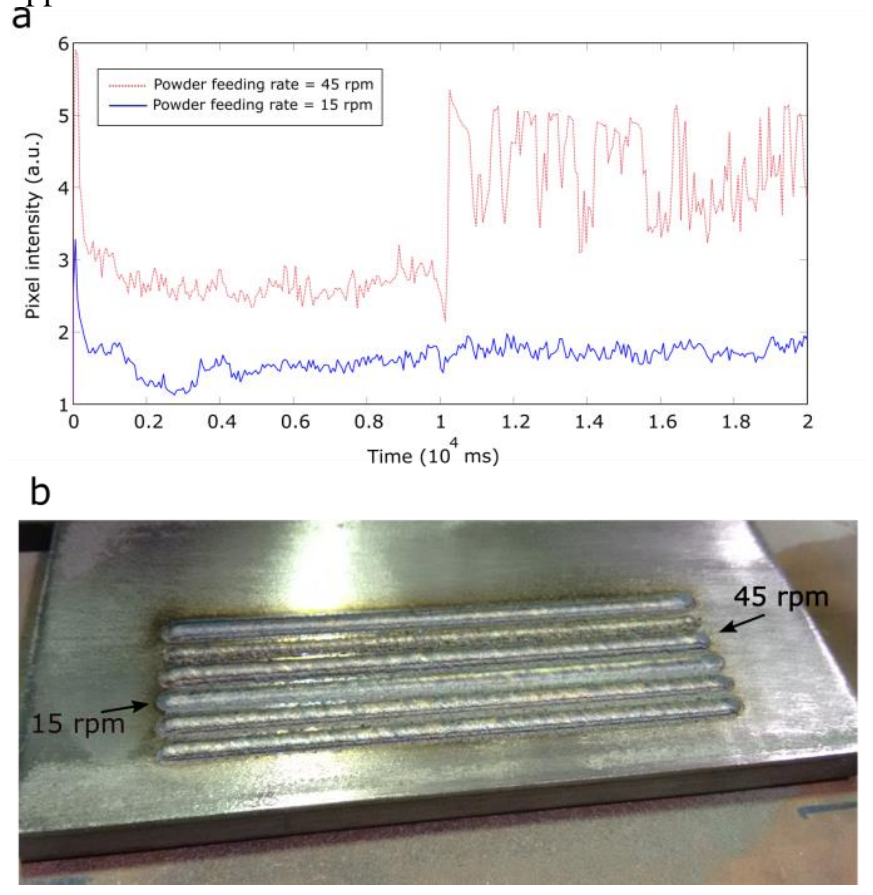

Figure 4. (a) Pixel ( $\# 1440, \lambda \approx 850 \mathrm{~nm}$ ) evolution for two clad tracks performed on AISI-316 with powder feeding rates of 15 (blue) and $45 \mathrm{rpm}$ (dotted line, red); (b) associated clad tracks.

A similar example is presented in Fig. 5 (a), where two consecutive clad tracks have been carried out on a QR0-90 substrate with different laser powers: 1000 and $1400 \mathrm{~W}$. The output profile was generated in this case by the plasma RMS signal [19]. The two signals show different levels, and the passes of the laser beam ( 2 clad tracks, four passes) can also be clearly appreciated.

\section{SPECTRAL PROCESSING BASED ON NORMALIZATION AND CORRELATION TECHNIQUES}

The previous experimental tests were based on perturbations that create clear differences in the process state. A more subtle experiment was designed by changing the powders used during the process. 3 different powders were used: Maraging, P44 and PV4. The composition of the latter is unknown, as it is involved in a research project currently in progress. Compositions of Maraging and P44 are presented in Table 2.

TABLE II

COMPOSITIONS OF POWDERS MARAGING AND P44

\begin{tabular}{cccccccc} 
Powder & $\mathrm{C}$ & $\mathrm{Co}$ & $\mathrm{Mo}$ & $\mathrm{Ni}$ & $\mathrm{Ti}$ & $\mathrm{V}$ & $\mathrm{Fe}$ \\
& & & & & & & \\
$\begin{array}{c}\text { Maraging } \\
\text { P44 }\end{array}$ & 0.01 & 15.10 & 13.20 & 10.10 & 0.90 & - & Bal. \\
\hline
\end{tabular}

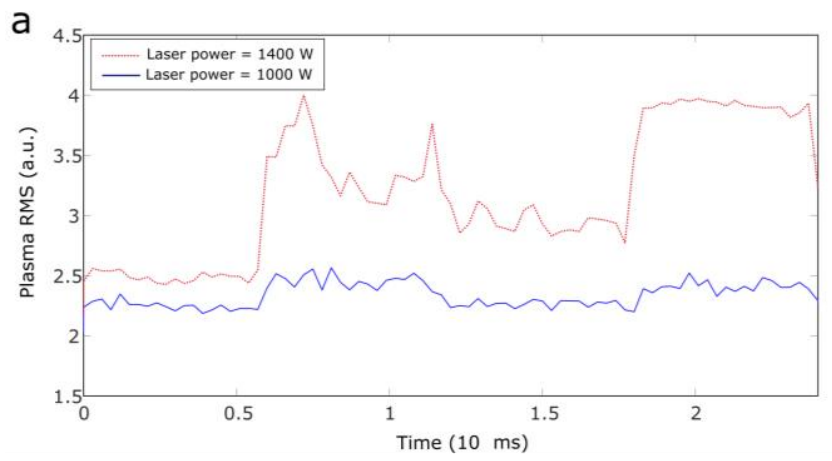

b

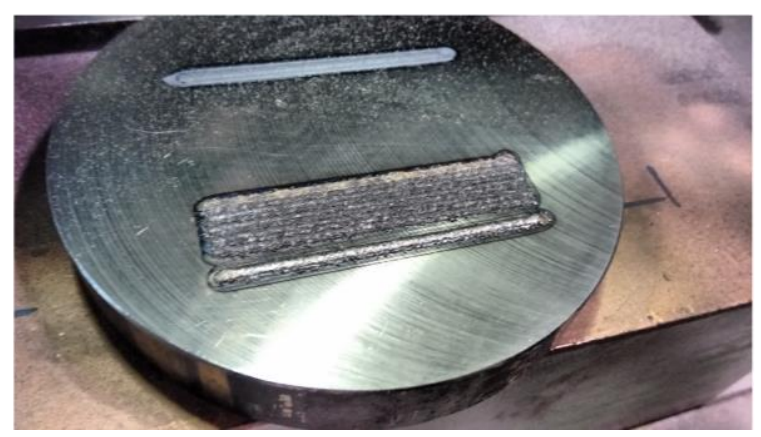

Figure 5. (a) Plasma RMS profile for two consecutive clad tracks performed on QR0-90 with laser powers of 1000 (blue) and $1400 \mathrm{~W}$ (dotted line, red); (b) associated clad tracks.

Based on the experience of TM Comas, the use of Maraging generates optimal clad tracks, while PV4 gives rise to some internal porosities and P44 more defects, such as porosities 
and cracks. An initial analysis was performed by using the plasma RMS signal as monitoring output parameter for LMD clad tracks performed with these three powders. Figure 5 shows a comparison of the plasma RMS signals for clad tracks performed using Maraging and P44 (Figure 6 (a)) and PV4 (Figure 6 (b)), respectively. It can be observed how noise (measured as the standard deviation of the signal) is clearly lower in both cases for Maraging, thus indicating a more stable process. Additionally, the average value of the plasma RMS signals is higher for P44 and PV4. An example of the resulting clad tracks is shown in Fig. 6 (c), with tracks performed with Maraging, P44 and PV4 from left to right.


Figure 6. (a) Comparison of plasma RMS profiles for clad tracks performed on QR0-90 with powders Maraging (red) and P44 (blue); (b) comparison of plasma RMS profiles for clad tracks performed on QR0-90 with powders Maraging (red) and PV4 (blue); (c) associated clad tracks.

The ability of the system to detect variations on the powder/gas feeding/flow rates was also investigated. In the experiment discussed in Fig. 7, performed with Maraging, the shielding gas flow rate was derived from the initial nominal parameter as pressure was diminishing during the process by manipulating the associated tube. At the final stage $(t \approx 95 \mathrm{~s})$, the normal gas flow rate was recovered, while the powder feeding was completely blocked. All these perturbations can be detected in the monitoring signal profile, which has been compared to a nominal clad track.

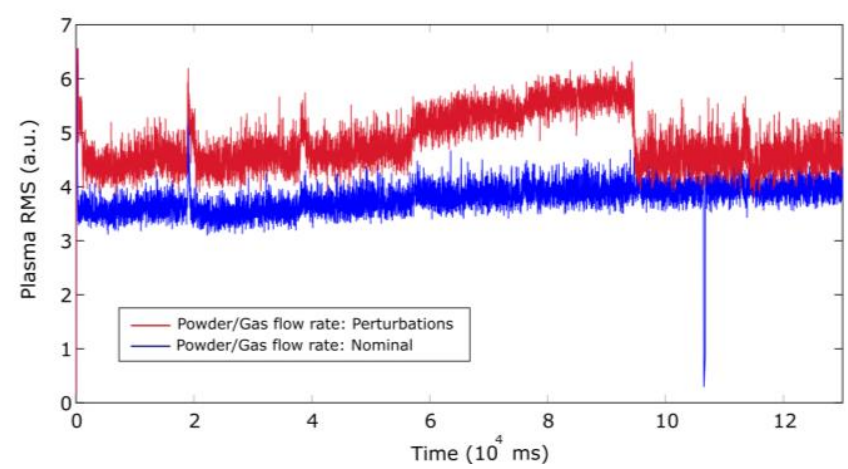

Figure 7. Comparison of plasma RMS profiles for clad tracks performed on QR0-90 with Maraging and nominal parameters (blue) and performing perturbations in the powder and gas flow rates (red).
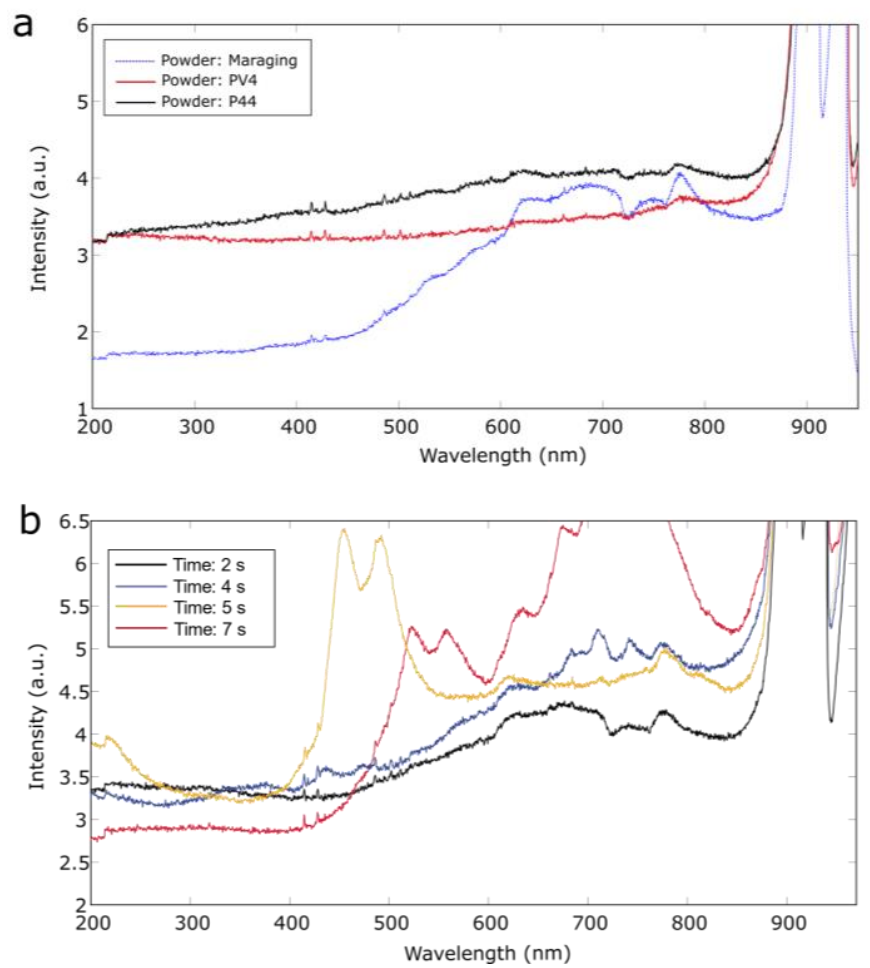

Figure 8. Plasma spectra captured with spectrometer ECONIC: (a) spectra associated with clad tracks performed with different powders; (b) spectra captured at different moments during a LMD test with powder PV4 and without predefined perturbations.

Although the plasma RMS signal seems to be useful in terms of detecting detective situations during the process, we decided to propose a different approach based on spectral correlation, in an attempt to find a more quantitative analysis tool. During the on-line monitoring of the spectra associated with the trials, we noticed that the shape of these spectra, i.e. 
the background radiation, significantly changed according to the applied perturbation.

Figure 8 (a) shows for example a comparison of spectra associated with the 3 selected powders during LMD trials performed with their corresponding nominal parameters. Clear differences can be observed in the morphology of the acquired spectra, particularly between Maraging and the other two. Spectra associated with a single LMD trial are presented in Fig. 8 (b): the clad track was performed with powder PV4 and without predefined perturbations. Clear differences can be observed in the associated spectra, thus indicating the lack of stability of the process. Changes in the continuum radiation have been related to the energy input of the process by several authors. In a LMD process, the contribution of the blackbody radiation may originate from the melt pool or from the plasma plume generated in the laser-powder interaction [8], and other factors such as the vapor absorption or the disposition of the input optics will also affect the acquired spectra. The mentioned changes in the spectra of Fig. 8 (b) are consistent with the high rate of defects derived from the use of PV4, as will be discussed at the end of this section.

A first step to perform the analysis based on correlation is to determine the reference spectrum for a given LMD process scenario, i.e. nominal parameters and base and powder materials. It is generated as follows:

1. A region of interest (ROI) is determined, for example excluding the spectral region associated with the laser radiation.

2. All the spectra of a certain nominal process are averaged, discarding some spectra associated with the beginning and end of the process. An initial reference spectrum is calculated.

3. The spectra showing a correlation higher than 0.8 to the previously calculated reference spectrum are selected and the final reference spectrum is determined with the same averaging technique.

There are different approaches to carry out the correlation analysis, however, a normalization stage is required if scaling or gain effects are to be avoided. The multiplicative scatter correction (MSC) technique has been chosen in this case to perform the normalization of the spectrum under analysis [20]. Basically, it performs a correction in the recorded spectra by changing their scale and offset, using a reference spectrum in the process. In addition to this normalization, a spectral region of interest (ROI) is defined to choose the suitable spectral range to be considered.

Following this procedure, Fig. 9 shows the result of applying this technique to the clad tracks performed with Maraging and already analyzed in Fig. 6 (a) and Fig. 7. Fig. 9 (a) presents the correlation error (where 0 denotes total correlation and 1 complete lack of correlation) associated with a clad track performed with nominal parameters and without predefined perturbations.
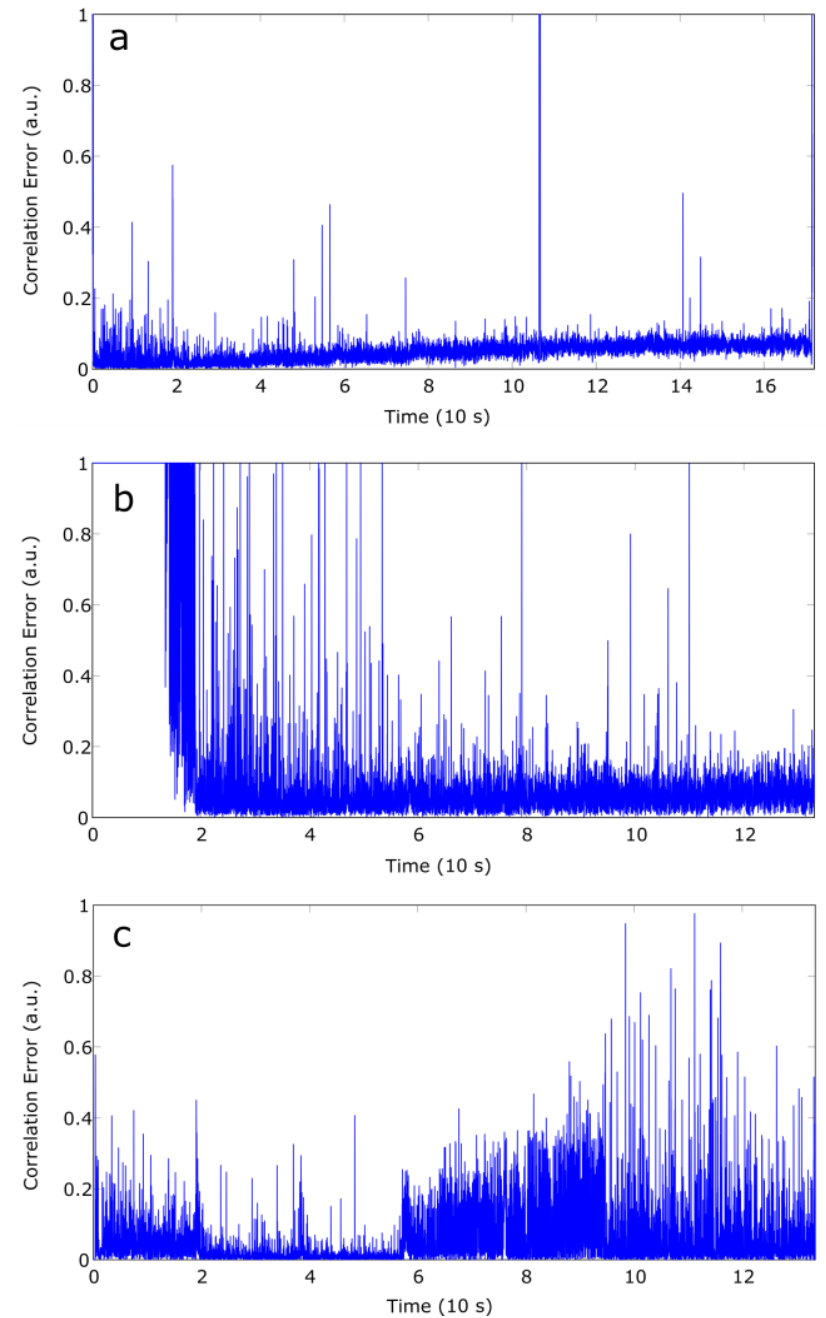

Figure 9. Correlation analysis of clad tracks performed with powder Maraging: (a) nominal parameters; (b) deviation of the laser focal point; (c) perturbations on powder and gas feeding/flow rates.

Although the average correlation error $(0.05)$ is low, several peaks indicate the appearance of possible defects. This situation will be discussed later with Fig. 11. Predefined perturbations were introduced in the tests show in Fig. 9 (b) and (c). A deviation from the original laser spot position on the cylinder (base material) was performed in the former, while both gas and powder flow/feeding rates were modified in the latter. The average correlation error in Fig. 9 (b) is 0.24 , while the sections associated with the gas flow rate ( $\mathrm{t} \approx 6$ to 10 $\mathrm{s}$ ) and powder feeding rate ( $\mathrm{t} \approx 10$ to 13.3 s) clearly exhibit higher correlation errors.

The use of this analysis with any of the tests performed with powders P44 or PV4 gives rise to very noise profiles, thus confirming the lack of stability observed during the process. An example is presented in Fig. 10, where results associated with clad tracks carried out with P44 are shown. Fig. 10 (a) is associated with a P44 clad track with nominal parameters, while Fig. 10 (b) shows the result of the same experiment, but considering a pre-heating stage $\left(400^{\circ} \mathrm{C}\right)$ of the base material (see Fig. 10 (c)), which is supposed to alleviate the occurrence of cracks [21]. In both cases the resulting profiles are very noisy, with associated standard deviations of 0.228 (Fig. 10 
(a)) and 0.194 (Fig. 10 (b)), in comparison to 0.05 of Fig. 9 (a). The average correlation error is consistent with these considerations: 0.72 and 0.31 , respectively.
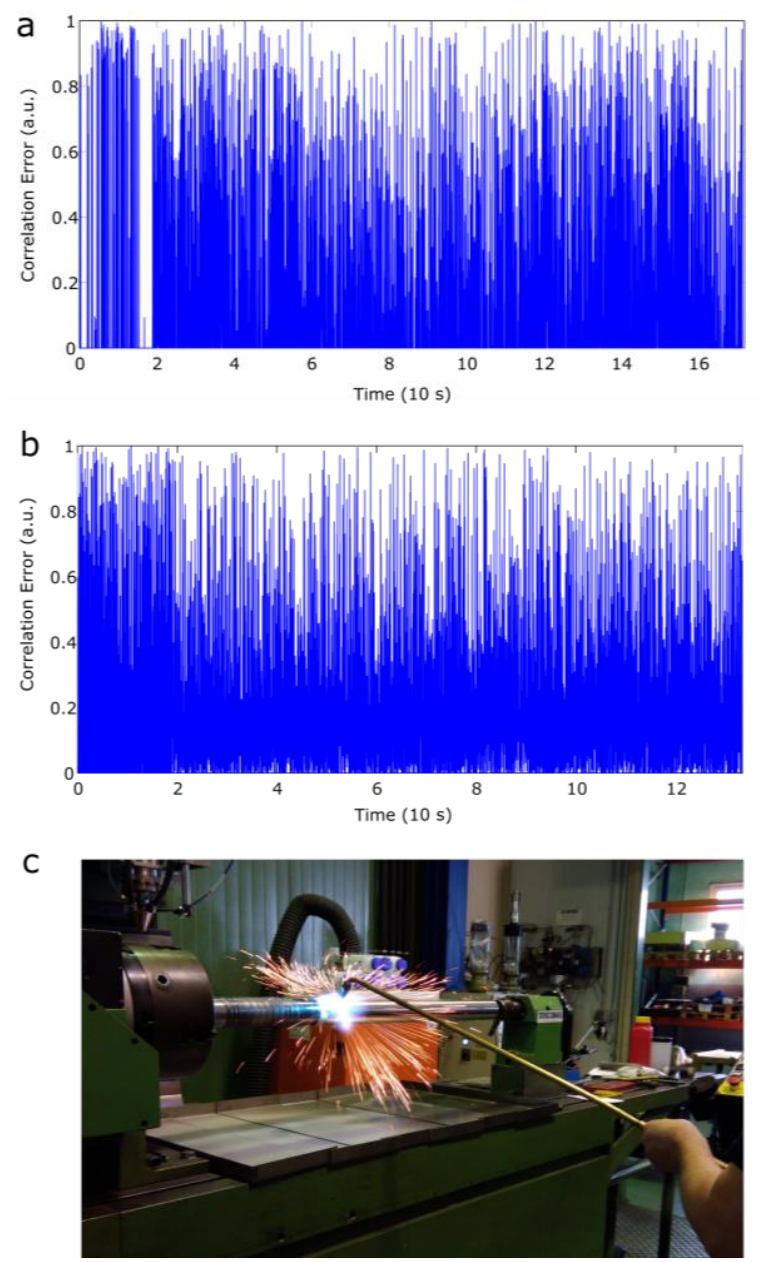

Figure 10. Correlation analysis of clad tracks performed with powder P44: (a) nominal parameters; (b) clad track performed after pre-heating stage $\left(400^{\circ} \mathrm{C}\right) ;(\mathrm{c})$ image of the preheating stage.

Considerations about the performance and final quality associated with the use of Maraging, PV4 and P44 powders have been backed by the knowledge of the staff of TM Comas, as well as by visual inspections performed after the trials. Macrographs, depicted in Fig. 11, also support the previous comments. Macrographs of clad tracks performed with Maraging (Fig. 11 (a)), PV4 (Fig. 11 (b)), and P44 without (Fig. 11 (c)) and with pre-heating (Fig. 11 (d)) are presented. Maraging gives rise to small porosities, which might explain the peaks appearing in the correlation error profile in Fig. 9 (a). Larger porosities can be observed associated with PV4 in Fig. 11 (b), while small porosities but also cracks can be observed in Fig. 11 (c). The use of the pre-heating stage seems to avoid the appearance of cracks with $\mathrm{P} 44$, although porosities are still present.
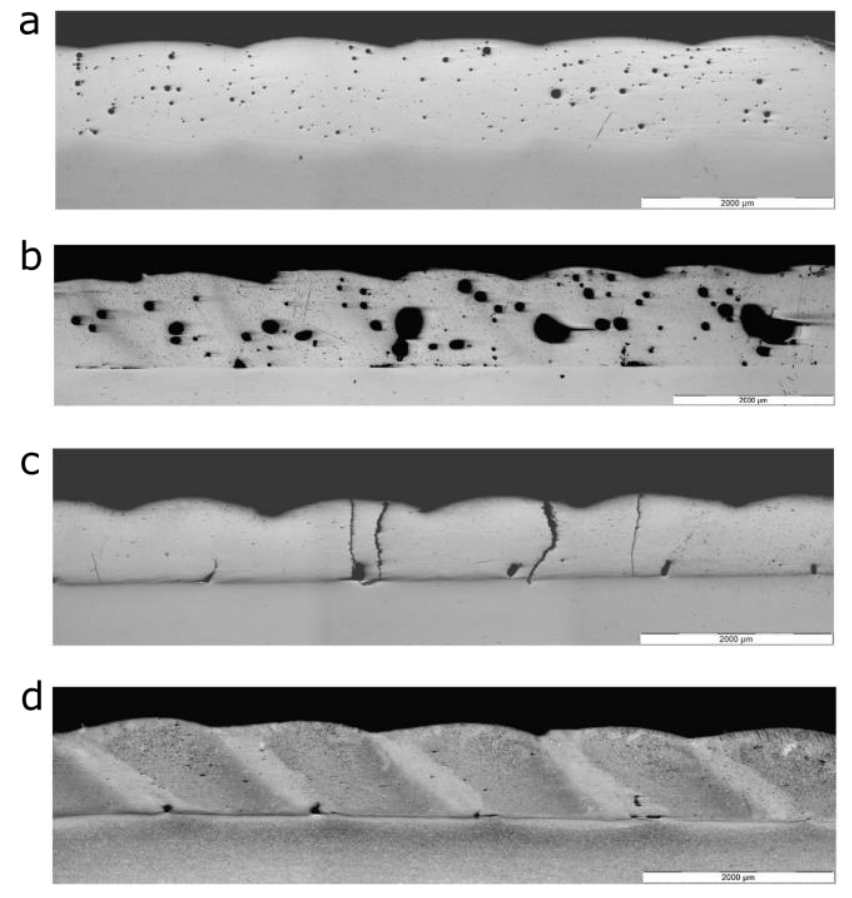

Figure 11. Macrographs associated with clad tracks performed with different powders: (a) Maraging; (b) PV4; (c) P44; (d) P44 with preheating stage.

\section{CONCLUSIONS}

On-line monitoring of a LMD process via plasma emission spectroscopy has been discussed in this paper. While traditional spectroscopic approaches are based on the identification and use of plasma emission lines for the generation of output monitoring parameters, we have analyzed the feasibility of alternative solutions based on the study of the plasma background emission. Simple approaches such as the plasma RMS signal have been evaluated, showing that predefined perturbations like variations in the gas or powder flow/feeding rates can be detected in the resulting profiles. A morphological analysis of the plasma background radiation, based on the estimation of a reference spectrum and the use of the MSC technique and correlation analysis, has also been proposed. The obtained results, where the effect on the final quality of three different powders have been studied, are in agreement with the visual inspection of the clad tracks and the resulting macrographs, showing the feasibility of a more quantitative quality monitoring approach. The performed trials have allowed to validate the feasibility of this proposal to be used as a viable monitoring solution. However, future works focused on developing more trials enabling the generation of a set of clad track defects will be required to better evaluate the performance of the proposed solution. In this regard, it would be necessary to obtain clad tracks where more specific (point) defects could be detected. As these specific defects (pores, cracks, contamination, etc.) give rise to perturbations in the associated spectra $[13,22,23]$, the proposed technique is expected to be able to detect them. In addition, the use of non-destructive techniques will help to evaluate the benefits of this spectroscopic approach. Analysis of alternative error metrics will also be considered. 


\section{REFERENCES}

[1] M. Ebo and A. E. Lindemanis, "Advances in Laser cladding Technology," in Proc. ICALEO, SPIE 527, Los Angeles, USA, 1983, pp. 86-94.

[2] G. Bi, A. Gasser, K. Wissenbach, A. Drenker, and R. Poprawe, "Identification and qualification of temperature signal for monitoring and control in laser cladding," Opt. Lasers Eng., vol. 44, pp. 1348-1359, Dec. 2006.

[3] L. Song, and J. Mazumder, "Feedback control of melt pool temperature during laser cladding process," Control Syst. Technol. IEEE Trans. On, vol. 19, pp. 1349-1356, Nov. 2011.

[4] J. T. Hofman, B. Pathiraj, J. van Dijk, D. F. de Lange, and J. Meijer, "A camera based feedback control strategy for the laser cladding process," $J$. Mater. Process. Technol, vol.. 212, pp. 2455-2462, Nov. 2012.

[5] L. Song, C. Wang, and J. Mazumder, "Identification of phase transformation using optical emission spectroscopy for direct metal deposition process," In High Power Laser Materials Processing: Lasers, Beam Delivery, Diagnostics, and Applications 8239, San Francisco, USA, 2012, p. 82390G.

[6] M. Ferrara, A. Ancona, P. M. Lugara, and M. Sibilano, "Online quality monitoring of welding processes by means of plasma optical spectroscopy", in High-Power Lasers in Manufacturing, Osaka, Japan, pp. $750-758$, Feb. 2000

[7] A. Ancona, V. Spagnolo, P. M. Lugara, and M. Ferrara, "Optical sensor for real-time monitoring of $\mathrm{CO} 2$ laser welding process," Appl. Opt., vol. 40, pp. 6019-6025, Nov. 2001.

[8] K. Bartkowiak, "Direct laser deposition process within spectrographic analysis in situ," Physics Procedia, vol. 5, pp. 623-629, Aug. 2010.

[9] L. Song, and J. Mazumder, "Real time $\mathrm{Cr}$ measurement using optical emission spectroscopy during direct metal deposition process," IEEE Sensors Journal, vol. 12, pp. 958-964, May 2012.

[10]L. Song, W. Huang, X. Han, and J. Mazumder, "Real-time composition monitoring using support vector regression of laser-induced plasma for laser additive manufacturing," IEEE Transactions on Industrial Electronics, vol. 64, pp. 633-642, Jan. 2017.

[11]W. Ya, A. R. Konuk, R. Aarts, B. Pathiraj, and B. Huis, B., "Spectroscopic monitoring of metallic bonding in laser metal deposition," Journal of materials processing technology, vol. 220, pp. 276-284, Feb. 2015.

[12] C. B. Stutzman, A. R. Nassar, and E. W. Reutzel, "Multi-sensor investigations of optical emissions and their relations to directed energy deposition processes and quality," Additive Manufacturing, vol. 21, pp. 333-339, May 2018

[13] B. Chen, Y. Yao, C. Tan, Y. Huang, and J. Feng, "A study on spectral characterization and quality detection of direct metal deposition process based on spectral diagnosis," The International Journal of Advanced Manufacturing Technology, pp. 1-11, March 2018.

[14] A. J. Dunbar, and A. R. Nassar, "Assessment of optical emission analysis for in-process monitoring of powder bed fusion additive manufacturing," Virtual and Physical Prototyping, vol. 13, pp. 14-19, Oct. 2017.

[15] T. Sibillano, A. Ancona, V. Berardi, E. Schingaro, P. Parente, P. M. Lugara, "Correlation spectroscopy as a tool for detecting losses of ligand elements in laser welding of aluminium alloys," Opt. Lasers Eng., vol. 44, pp. 1324-1335, Feb. 2006

[16] J. Mirapeix, A. Cobo, S. Fernandez, R. Cardoso, and J. M. LopezHiguera, "Spectroscopic analysis of the plasma continuum radiation for on-line arc-welding defect detection," J. Phys. D, vol. 41, pp. 135202 135210, June 2008.

[17] J. Mirapeix, R. Ruiz Lombera, J. J. Valdiande, A. Cobo, J. M. LopezHiguera, "Colorimetric analysis for on-line arc-welding diagnostics by means of plasma optical spectroscopy," IEEE Sensors Journal, vol. 16, pp. 3465-3471, May 2016.

[18] A. Kisielewicz, F. Sikström, A. K. Christiansson, and A. Ancona, "Spectroscopic monitoring of laser blown powder directed energy deposition of Alloy 718," Procedia Manufacturing, vol. 25, pp. 418-425, May 2018

[19] J. Mirapeix, A. Cobo, J. Fuentes, M. Davila, J. M. Etayo, and J. M. Lopez-Higuera, "Use of the plasma spectrum RMS signal for arc-welding diagnostics," Sensors, vol. 9, pp. 5263-5276, July 2009.

[20] H. Martens, and T. Naes, Multivariate calibration. Ed. Oslo: John Wiley \& Sons, pp. 237-266, 1989

[21] A. García de la Yedra, M. Pfleger, B. Aramendi, M. Cabeza, F. Zubiri, T. Mitter, ... \& E. Scherleitner, "Online cracking detection by means of optical techniques in laser- cladding process," Structural Control and Health Monitoring, vol. 26, pp. e2291, 2019.

[22] W. W. Liu, Z. J. Tang, X. Y. Liu, H. J. Wang, and H. C. Zhang, "A review on in-situ monitoring and adaptive control technology for laser cladding remanufacturing,". Procedia Cirp, vol. 61, pp. 235-240, 2017.

[23] A. R. Nassar, T. J. Spurgeon, and E. W. Reutzel, "Sensing defects during directed-energy additive manufacturing of metal parts using optical emissions spectroscopy," In Solid Freeform Fabrication Symposium Proceedings, Austin, Texas, pp. 278-287, Aug. 2014.

Jose J. Valdiande obtained his Telecommunication Technical Engineering Degree in 2003 and his Telecommunication Engineering Degree in 2012. He has been a member of the Photonics Engineering Group of the University of Cantabria since 2003 working in industrial and R\&D projects.

His main work is focused on real time monitoring for welding processes based on spectroscopy. In this regard, he is Co-founder of "SADIQ Engineering", a company offering products and services for welding, automation and monitoring. Also, he is co-founder of "eDrónica, technology for unmanned vehicles", a company focused on both aerial and underwater unmanned vehicles, recognized as a technology-based company according to ANCES and also awarded with entrepreneurship awards.

$\mathrm{He}$ is the co-author of more than a dozen articles in magazines and contributions to conferences. He is also coauthor of 7 national and international invention patents.

Jesus Mirapeix (Master Degree in Telecommunications) got his $\mathrm{PhD}$ in 2007 with a thesis focused on the use of plasma optical spectroscopy in the quality monitoring of welding processes. This work was recognized as the "best thesis in engineering" in the 2009 edition of the "Premios de Investigación del Consejo Social" (Universidad de Cantabria).

Since 2012 he has been working as Associate Professor in the Photonics Engineering Group of the University of Cantabria, developing both teaching and researching tasks.

He has collaborated in research projects funded by the European Union (2), and by national entities (20). He has also participated in 9 research contracts with public and private companies. He is author/co-author of more than 30 papers in international journals ( $\mathrm{h}$ index $=14$ (Scopus), 16 (Google Scholar)), of more than 70 contributions to international conferences and has collaborated in 5 patents.

Nowadays his research interests are focused on distributed sensor systems based on Brillouin scattering and plasma emission spectroscopy.

He is co-founder of SADIQ Engineering, a spin-off devoted to the development of industrial monitoring systems based on photonic technology.

Jaume Nin is the responsible of $\mathrm{R}+\mathrm{D}$ and Technical department of TMCOMAS (Talleres Mecánicos Comas) since 2009. Chemist after University of Barcelona, he achieved his $\mathrm{Ph}-\mathrm{D}$ in materials science in 2005. After some briefs stages in leading thermal spray research centers in Tampere (Finland) and Montreal (Canada), he started his professional career in a hardfacing feedstock company for almost a year. Then he enrolled TMComas as a workshop manager until 2009. 
In the past he was the director of the Centre for Thermal Spraying of the University of Barcelona with project management functions and industrial production. $\mathrm{He}$ is a European/international welding engineer (IWE). He has vast experience in thermal spraying: more than 20 national and international publications. He has participated in structural characterization techniques of materials and collaborated in patents and books. He has been involved in several European and Spanish funded $\mathrm{R}+\mathrm{D}$ projects, acting as a Partner technical coordinator.

He has also a vast experience in rotor repair techniques, material science, metallurgy, heat treatments, surface engineering techniques, vacuum brazing,

Eloi Font obtained the Degree in Mechanical Engineering in 2011 at Universitat de Girona and also a Master Degree in Design of Metallic Components at Universitat Politecnica de Catalunya. He joined Talleres Mecánicos Comas (Blanes, Spain) in 2013, where he works in the quality monitoring of welding and laser metal deposition processes.

Carlos Seijas Albir got his Degree in Mechanical Engineering in 2011 and a master in bodyworks and automotive materials in 2013. Since then, he has been always working in the automobile sector, starting his career in the company SEAT (VW GROUP) and moving to GESTAMP AUTOMOTIVE in 2015 where he currently works as a Process and project engineer in the Research and development center the company has in Shanghai (China).

During the last 6 years in GESTAMP AUTOMOTIVE, Carlos has specialized in the development and innovation of manufacturing technologies for body in white components.

José-Miguel López-Higuera (M'93-SM'98) was born in February 1954, in the village of Ramales de la Victoria, Cantabria, Spain. He obtained the Telecommunication Technical Engineering degree from the Universidad Laboral de Alcala de Henares, Madrid, Spain and the Telecommunication Engineering degree from the Universidad Politécnica de Madrid (UPM), Madrid, Spain. He received the Ph.D. degree in telecommunication engineering, with an extraordinary award, from UPM. He founded and is the Head of the Photonics Engineering Group of the TEISA Department in the University of Cantabria. Currently, he works in the development of Photonics Instrumentation, photonic/optical fibre sensor systems for civil engineering, electrical power, environmental and smart structures and for optical diagnostics for a wide range of applications. He has directed more than 50 $\mathrm{R} \backslash \& \mathrm{D}$ projects and has written or co-written more than 400 publications in the form of books, chapters of books, papers and conferences, both national and international, and obtained 1 ten patents. He is the editor and co-author of several books, including i) Optical Sensors (UC, 1998); ii) the Handbook of Photonic Sensing Technology (Wiley, 2002) and iii) he is the co-editor of the book Engineering a High-Tech Business: Entrepreneurial Experiences and Insights (SPIE-Press, 2008). Prof. Lopez-Higuera is Senior Member of the IEEE' and member of the IEE, SPIE and OSA. 